freely from Europe (chiefly Great Britain and France) and more lately from America, and have consequently found themselves faced with the duty of finding large amounts of interest. This interest often is not by any means earned by the working of the schemes on which the money was spent. To meet this call for interest, exports must largely exceed imports, and so tariffs are introduced to keep down imports, and local industries are started. On all sides, in the first great burst of mass- production, local boundaries seemed to have been swept away. It is probable that our social thoughts and plans will have to regain contact with mother earth, each group basing itself on its own soil, but evidently not in the old sense of a self-contained isolation. Interdependence of all on eack is a new feature that will become increasingly important, and one of the geographer's tasks is to try to see both the roots of each society in its own soil, and its relations to others.

\title{
White Dwarf Stars*
}

$\mathrm{I}^{\mathrm{N}}$ this year's Halley Lecture, Prof. E. A. Milne presents us with a systematic account of whito dwarf stars in theory and observation. There is no doubt that, in view of their peculiar physical properties and the excellent examiple they provide for a successful application of modern quantum mechanies, white dwarfs are among the most interesting of natural objects. But this interest arises, of course, not so much from the direct observations as from the derived density of the star and its theoretical interpretation. It is therefore very valuable, at least to the non-astrophysical scientific worker, to have the facts and theories about white dwarf stars reviewed in this systematic way.

Owing to the importance of the existence of matter of great density to general physical theory, it is important to appreciate how direct is the evidence and how independent of any detailed theory. The well-established white dwarfs like Sirius B have a mass directly deduced from the law of gravitation and an observed double star orbit. They have an absolute luminosity determined from their distance (parallax) and their observed apparent luminosity. In view of the simplicity and universality of the theories involved in these deductions, no one will dispute that mass and absolute luminosity have the certainty of direct observations. To determine a density, a radius is required as well as a mass, and the radius must be deduced from the absolute luminosity and the surface temperature via the thermodynamic laws of radiation and the theory of the flux of radiation emerging from a gaseous atmosphere. Even in this step the maximal observational and theoretical uncertainties are not very great. If we know the surface temperature and the absolute luminosity, we can determine the surface area of the star, apart from possible uncertainties, at most of the order of 50 per cent, arising from conceivable errors in the radiative theory. All depends, therefore, on a determination of surface temperature, to which parameter the resulting mean density is rather sensitive, as it varies as the sixth power of this temperature.

There are two main methods of determining surface temperature. One can assume that the quality of stellar radiation, apart from line absorptions, follows closely Planck's black body curve ; after observing the energy distribution in the

" "The White Dwarf Stars" being the Galley Lecture delivered on May 19 1932, by Prof. E. A. Milde. (Oxford: Clarendon Preso. 1032.) No. 3286 , Vol. 130$\rfloor$ spectrum of the star, one can fit Planck curves to it and thereby determine the temperature from the curves of best fit. This method is known to underestimate the temperature considerably, especially for the higher surface temperatures, and is not reliable for the stars in question. But the theory of ionisation in stellar atmospheres and of the production of absorption lines in an ionised atmosphere enables one to assign a surface temperature when the surface value of gravity is known and also the spectral type. The possible resultant uncertainties in this application of theory do not. appear to be very great. It is true that the surface value of gravity is not known initially, but it is known when the radius is determined, and the problem of determining the surface temperature is therefore solvable in theory, and also in practice, at worst by a process of successive approximation. This is admittedly the most uncertain step in the deduction of the radius and therefore of the density, but if we suppose that a maximum uncertainty of a factor of 10 eould thereby be introduced into the density, we shall have allowed more than amply for all errors at present conceivable, and the existence of excessively dense matter must be regarded as fully established. Taking a reasonable view of probable observational and theoretical uncertainties, it is most unlikely that the current accepted mean densities for the best determined white dwarfs, such as Sirius B, are in error by a factor nearly so large.

Prof. Milne then passes on to the problem of the frequency of such dense matter in our galaxy. There are very few directly well observed white dwarfs, but the conditions for a good determination, owing to absolute faintness and the necessity for a well determined parallax and double star orbit, are very severe, and the fact that we know only four such stars is consistent with a great abundance of such stars in our galaxy. The essential conditions for a white dwarf are a relatively low absolute luminosity combined with a high surfaco temperature. There is reason to believe that these properties are found together in two remarkable classes of stars, the central stars of planetary nebulæ and ex-novæ. The evidence is reviewed by Milne, who concludes provisionally that the case is proved.

If correct, this conclusion is of the utmost importance, not so much for the greatly increased abundance of known dense objects as for its possible 
evolutionary significance. It has been suggested by Prof. Milne, on purely theoretical grounds, that stars in general are in one of two states which may be called expanded and collapsed (Milne's centrally condensed and collapsed configurations respectively), and that the passage from one state to the other sets in at a certain critical stage in the evolutionary history and is of a cataclysmic nature. It is suggested, in short, that the evolving star, perhaps towards the end of its radiating life, becomes a nova and then a white dwarf. The outburst of radiation during the transient nova stage is the star's means of getting rid of the great difference of energy between the contiguous expanded and collapsed states. While it is still too early to be confident of the correctness of the theory underlying Milne's suggestion, the suggestion itself is a very happy one, likely-right or wrong-to prove extremely fertile. Milne's discussion already shows that an evolutionary sequence, ordinary star -nova-white dwarf, is by no means irreconcilable with the known facts, and indeed co-ordinates a great part of the facts in a most satisfying manner.

The second half of the Halley Lecture describes the present state of knowledge of the theory of stellar configurations-the generalisations that have sprung in the last three years from Eddington's pioneer work on the radiative equilibrium of a sphere of perfect highly ionised gas. These generalisations are still in an early stage of development. Milne himself, who initiated them and has been the prime mover in their development, would, I believe, be the last to claim that the equilibrium states of any model yet computed really reproduce the properties of any ordinary star. The collapsed white dwarf states appear to be simpler and to be satisfactorily accounted for by models already computed. They consist mainly of a rather cool, dense, almost isothermal core, the properties of which are controlled by those of a degenerate electron gas. But although existing models have not yet been made to mimic the observed properties of ordinary stars, we can see that this study of models has already infused new life into the theories of stellar evolution, and has taken us perhaps one long step nearer to the goal so rashly proposed as attainable in the last sentence of Eddington's " Internal Constitution of the Stars"-the goal of understanding so simple a thing as a star.

\section{R. H..F}

\section{The British Association Standards of Electrical Resistance, I862-I932}

\section{F} EW realise how much the electrical industry owes to the Electrical Standards Committee of the British Association, first appointed at the Manchester meeting in 1861 . We welcome, therefore, the paper entitled "Material Standard of Resistance : the B.A. Coils, 1881-1932 ", read by Sir Richard Glazebrook and Dr. Hartshorn to Section A (Mathematical and Physical Sciences), at the York meeting on September 1. The Electrical Standards Committee in its first report (Cambridge, 1862) stated that it had first to determine the most convenient unit of resistance and, secondly, the best form and material for the standard representing that unit. The C.G.S. system of measurement was the outcome of its deliberations, and this decision has done more than perhaps any other single act in simplifying and unifying electrical measurements throughout the world.

Experiments were made at King's College, London, by Maxwell and Fleeming Jenkin to obtain the ohm in a material form, and reports giving their results were issued in 1863 and 1864. In Appendix A of the Report for 1865 it was recommended that the material of the wire from which the resistance standard be made should be an alloy containing 66 per cent silver and 33 per cent platinum. It was agreed that copies of the standard should be made and preserved at Kew Observatory. In the Report for 1867 a table is given of the values of the standards in question. This Committee was dissolved in 1870. Soon after Maxwell's appointment as Cavendish professor of experimental physics at Cambridge, the coils were brought to the Cavendish Laboratory and were used by Chrystal in 1876. Lord Rayleigh succeeded Maxwell in 1879 and at once became interested in electrical measurements. Rowland of Baltimore had thrown doubts on the accuracy of the absolute measurements of the B.A. Committee. Rayleigh and Schuster's experiments at Cambridge confirmed these doubts, and from 1881 onwards great activity was shown at the Cavendish Laboratory in investigating the question of electric units. In $1879-81$, Dr. J. A. Fleming made a very careful comparison of the B.A. coils. He found that their relative values had changed appreciably, and he adopted as a definition of the B.A. unit the mean value of the resistance found from all the coils at the temperatures at which they were originally said to be correct.

The Electrical Standards Committee was reappointed in 1880; and in 1881, R. T. Glazebrook became connected with the work. He was appointed secretary in 1883 , and the coils were in his charge from that date until 1919. They are still at the National Physical Laboratory. The Standards Committee was dissolved in 1912. Up to that date, comparisons of the coils between themselves were continually in progress, and their values were determined in ohms and also in terms of the length of a column of mercury by Rayleigh, Glazebrook, Fitzpatrick, and F. E. Smith. The records show that most of the coils have changed appreciably during their long life, but that the two platinum coils included in the original group have remained unchanged.

The point of most importance that emerged from Sir Frank Smith's measurements of 1908 was the performance of the two platinum coils, now marked ' $\mathrm{D}$ ' and ' $\mathrm{E}$ '. The value of these coils in B.A. units in 1888,1908 , and 1932 obtained from a comparison

No. 3286, Vor. 130] 